\title{
Prompt Photon Production in Deep Inelastic Scattering and Photoproduction at HERA
}

\section{Krzysztof NOWAK*}

Physik-Institut der Universität Zürich

E-mail: knowak@mail.desy.de

Three recent measurements of prompt photon production performed by the H1 and ZEUS collaborations using HERA ep collider data are presented. The prompt photon rates measured by $\mathrm{H} 1$ and ZEUS in deep inelastic scattering are compared to QCD calculations and to MC predictions. A preliminary $\mathrm{H} 1$ measurement in photoproduction is presented and results are compared to NLO collinear calculation and the calculation based on a $k_{T}$-factorisation approach.

European Physical Society Europhysics Conference on High Energy Physics

July 16-22, 2009

Krakow, Poland

${ }^{*}$ Speaker. 


\section{Introduction}

Isolated photons produced in deep inelastic scattering (DIS) and photoproduction together with the hadronic final state $X, e p \rightarrow e \gamma X$, so called prompt photons, are a powerful probe of the underlying dynamics. They generally require smaller corrections for hadronisation effects than measurements relying on jets. A good understanding of the Standard Model prompt photon production mechanism is also important for searches of new particles decaying into photons in hadron colliders.

\section{Prompt photon production in DIS}

There are two contributions to prompt photon production in DIS. Final state photons may originate from the quark ( $Q Q$ contribution) or may be emitted from the lepton ( $L L$ contribution). The interference contribution $(L Q)$ is expected to be small. The $Q Q$ part may arise from direct radiation of the photon from the quark or from quark-to-photon fragmentation of the quark. In the case of the studies presented below, the fragmentation part is suppressed by the photon isolation requirement.

Events with isolated photons in DIS have been measured [1] by the H1 collaboration. Isolated photons with transverse energy $3<E_{T}^{\gamma}<10 \mathrm{GeV}$ and in the pseudorapidity range $-1.2<\eta^{\gamma}<1.8$ are studied within the kinematic region $4<Q^{2}<150 \mathrm{GeV}^{2}$ and $y>0.05$ using an integrated luminosity of $227 \mathrm{pb}^{-1}$. Background from elastic Compton scattering has been suppressed by a cut on the invariant mass of the hadronic system $W_{X}>50 \mathrm{GeV}$. A photon candidate is identified as a cluster in the electromagnetic section of the calorimeter. Candidates with a track geometrically matching the cluster with a distance of closest approach of less than $20 \mathrm{~cm}$ are rejected. In order to suppress the background coming from neutral hadrons (predominantly $\pi^{0} \rightarrow \gamma \gamma$ ) the transverse radius of the cluster is required to be below $6 \mathrm{~cm}$. The isolation of the photon is ensured by requiring that it carries at least $90 \%$ of the transverse momentum of the jet containing the photon. The remaining neutral hadron contamination is estimated with a multivariate analysis statistically exploring the cluster shape differences between clusters originating from genuine photons and clusters produced by neutral hadron decay products.

The differential cross sections in bins of transverse energy of the photon $E_{T}^{\gamma}$, its pseudorapidity $\eta^{\gamma}$ and negative four-momentum transfer squared $Q^{2}$ are presented in figure 1 compared to leading order QCD calculation [2] and to predictions of the Monte Carlo models PYTHIA $(Q Q)$ and RAPGAP $(L L)$. Both predictions underestimate the data cross sections roughly by a factor of two, with the difference being the most significant at low $Q^{2}$.

New preliminary results from the ZEUS collaboration are reported as well. The results are based on data with integrated luminosity of $320 \mathrm{pb}^{-1}$ within the kinematic range of $10<Q^{2}<$ $350 \mathrm{GeV}^{2}$. Isolated photon candidates with transverse energy $4<E_{T}^{\gamma}<15 \mathrm{GeV}$ and in the pseudorapidity range $-0.7<\eta^{\gamma}<0.9$ are selected in the electromagnetic part of the calorimeter with no track assigned to it. The signal is extracted from the dominant neutral hadron background by a cluster shape analysis. The measured cross sections are compared to the two sets of calculations and to the Monte Carlo predictions. The leading order QCD calculation [2] (LO) is the full collinear calculation including $Q Q$ terms as well as $L L$ contributions. The MRST approach [3] calculates 

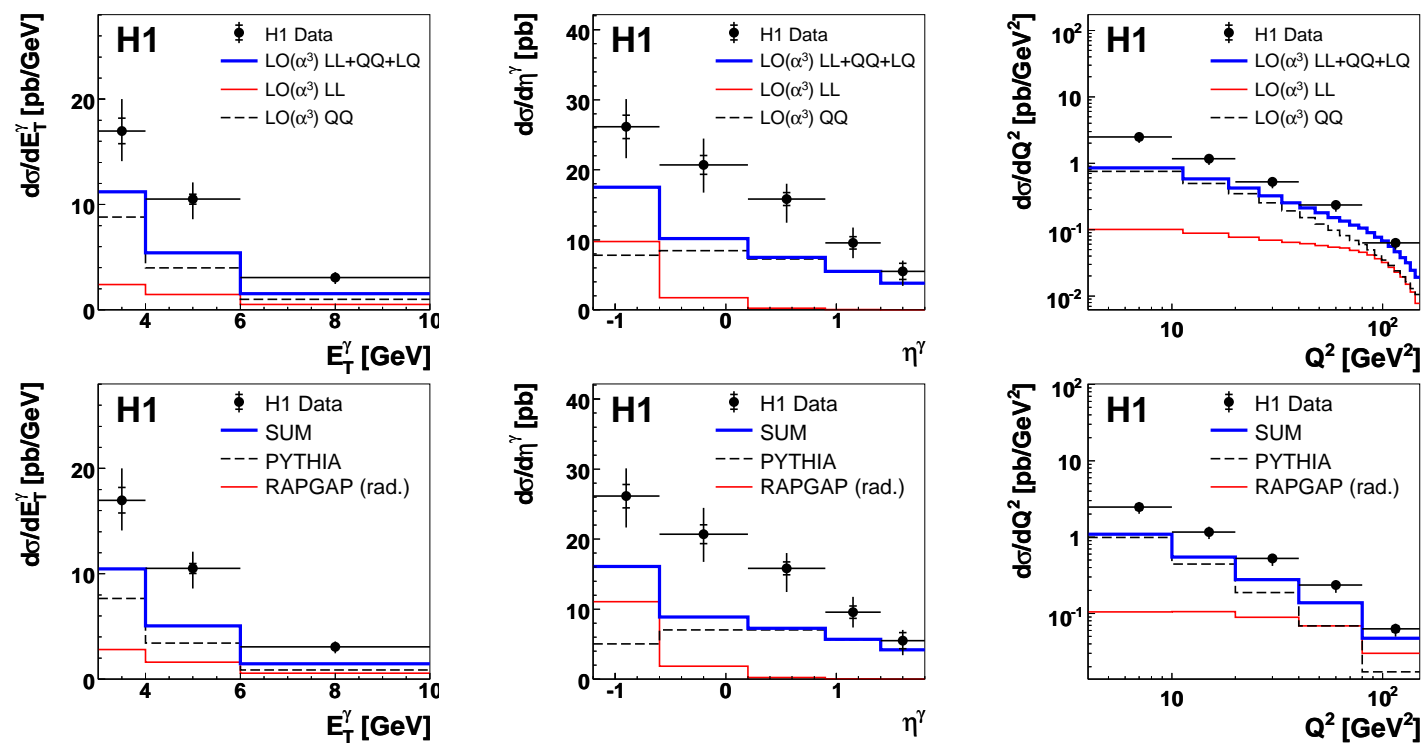

Figure 1: Differential prompt photon cross section in DIS compared to the LO calculation (top) and to the MC prediction (bottom) as measured by the $\mathrm{H} 1$ collaboration [1].
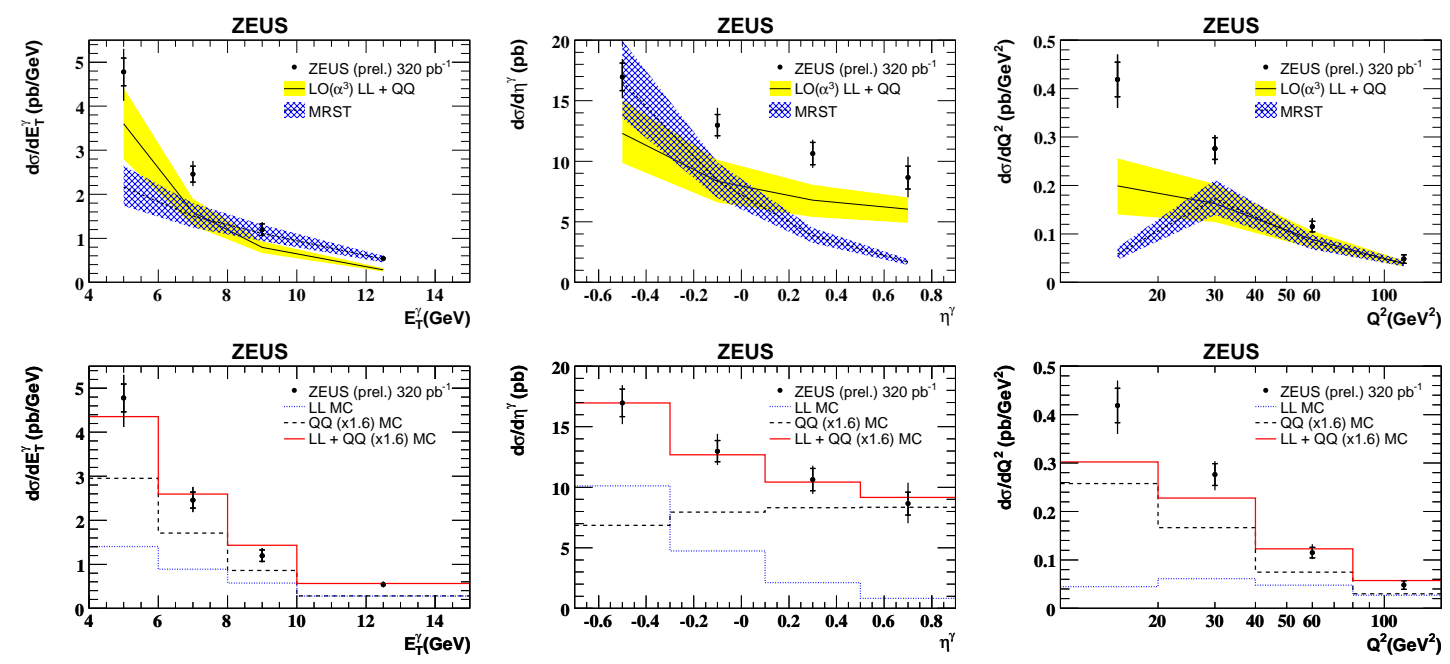

Figure 2: Differential prompt photon cross section in DIS compared to the calculations (top) and to the MC prediction (bottom) as measured by ZEUS collaboration.

the resummed version of the $L L$ contribution including the QED Compton scattering $\gamma_{p} e \rightarrow \gamma e$, where $\gamma_{p}$ is a constituent of the proton. At the same time its validity is limited due to the fact the $Q Q$ compontent was neglected. The MC PYTHIA model is used to model the $Q Q$ contribution together with DJANGOH for the $L L$ part.

Figure 2 presents the differential prompt photon cross sections in bins of $E_{T}^{\gamma}, \eta^{\gamma}$ and $Q^{2}$. Similar conclusions can be drawn with the LO calculation underestimating the experimental data most significantly at low $Q^{2}$. The MRST calculation agrees with the measurement at large $Q^{2}$ and low $\eta^{\gamma}$ where the $L L$ contribution is expected to dominate. MC models again underestimate prompt photon production and the $Q Q$ part needs to be scaled by a factor of 1.6 in order to properly 
describe the cross section.

A next-to-leading order calculation [ [ 4 is available for the production of a prompt photon in a conjunction with a jet. The hadronic jet is required to be found in the pseudorapidity range $-1.0<\eta^{j e t}<2.1$ and with the transverse energy above $2.5 \mathrm{GeV}$. The calculation was compared to the $\mathrm{H} 1$ measurement [1] and though higher than the leading order cross sections by roughly $20 \%$, it still significantly underestimates the measured cross sections.

\section{Prompt photon production in photoproduction}

The H1 collaboration reported preliminary results on a prompt photon cross section measurement in the photoproduction regime within the kinematic range given by $Q^{2}<1 \mathrm{GeV}^{2}$ and $0.1<$ $y<0.7$. The isolated photons with transverse energy $6<E_{T}^{\gamma}<15 \mathrm{GeV}$ and pseudorapidity $-1.0<\eta^{\gamma}<2.43$ are studied using data with $340 \mathrm{pb}^{-1}$ of integrated luminosity.

The photon candidates are defined as electromagnetic clusters with transverse radius below $6 \mathrm{~cm}$ and without associated signal in the $\mathrm{H} 1$ tracking devices. Low $Q^{2}$ DIS events are removed by excluding events with reconstructed scattered electron in the main $\mathrm{H} 1$ apparatus. It is additionally suppressed by removing events with inelasticity estimator $y_{h}$ being close to unity, where events with misidentified scattered electron accumulate. The DIS background is estimated to be below $1.5 \%$ in the final selection.
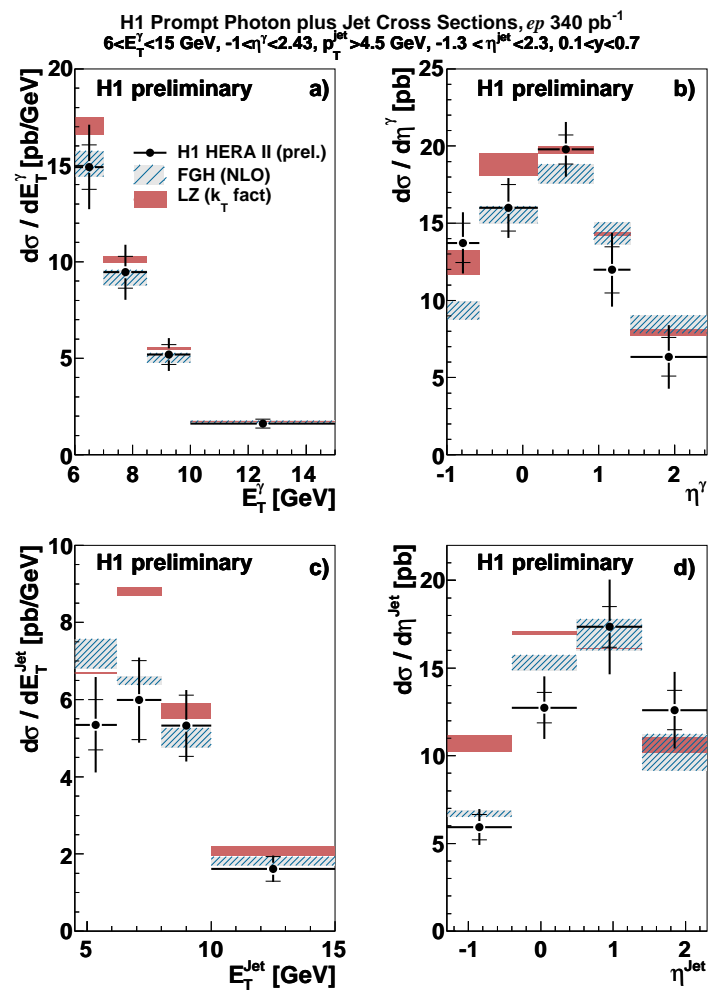

Figure 3: Preliminary differential prompt photon + jet cross section in photoproduction compared to the calculation predictions (see text) as measured by the $\mathrm{H} 1$ collaboration.

For the photon + jet case, the selection was based on the accompanying hadronic jet. Its transverse energy $E_{T}^{\text {jet }}$ is required to be above $4.5 \mathrm{GeV}$ and the jet itself must be found in the pseudorapidity range $-1.3<\eta^{\text {jet }}<2.3$.

Results are compared to a next-to-leading order collinear calculation (FGH) [5] and to a calculation based on the $k_{T}$-factorisation approach (LZ) [6]. Figure 3 presents the differential cross sections in bins of $E_{T}^{\gamma}, \eta^{\gamma}, E_{T}^{j e t}$ and $\eta^{\text {jet }}$ in the photon + jet case. Both calculations give a reasonable description of the $E_{T}^{\gamma}$ and $\eta^{\gamma}$, while only FGH calculation describes well $E_{T}^{\text {jet }}$ and $\eta^{\text {jet }}$ of the associated hadronic jet.

The correlations between the prompt photon and the hadronic jet were investigated by studying the cross sections in bins of the azimuthal acoplanarity between the photon and the jet $\Delta \Phi$, and 
the photon momentum perpendicular to the jet direction in the transverse plane $p_{\perp}$. Correlations are studied in the part of the phase space where direct events dominate $\left(x_{\gamma}^{L O}>0.8\right)$ and the supplementary one $\left(x_{\gamma}^{L O}<0.8\right)$ with enhanced contribution from resolved events. In this case $x_{\gamma}^{L O}$ denotes the estimator of the longitudinal momentum fraction of the parton in the photon entering the hard interaction and is defined as $x_{\gamma}^{L O}=E_{T}^{\gamma}\left(e^{-\eta^{j e t}}+e^{-\eta^{\gamma}}\right) /\left(2 y_{h} E_{e}\right)$, with $E_{e}$ being the electron beam energy. The results of the study are presented in figure 4 . Both predictions overestimate back-to-back configuration for the direct enhanced part of the phase space, but describe the tails reasonably well. The FGH calculation gives a reasonable description of the distributions for $x_{\gamma}^{L O}<0.8$, except for the highest bins in $p_{\perp}$ and $\Delta \Phi$. The region $\Delta \Phi \rightarrow 180^{\circ}$ is sensitive to multiple soft gluon radiation which limits the validity of fixed order calculations. The LZ calculation includes multiple soft gluon radiation in the initial state before the hard subprocess and describes $\Delta \Phi>170^{\circ}$ but predicts a significantly lower contribution of events in the tails of both distributions.

\section{Conclusions}

The measurement of prompt photons in $e p$ collisions remains an interesting topic. The new results highlight the deficits of the available QCD calculations. In DIS as well as in photoproduction, the available calculations are not able to describe all aspects of the data. More theoretical investigations are needed to understand the observed discrepancies.

\section{References}

[1] F. D. Aaron et al., Measurement of isolated photon production in deep-inelastic scattering at HERA, Eur. Phys. J. C54 371 (2008) [arXiv:0711.4578].

[2] A. Gehrmann-De Ridder et al., Isolated photons in deep inelastic scattering, Eur. Phys. J. C47 395 (2006) [hep-ph/0601073].

[3] A.D. Martin et al., Parton distributions incorporating QED contributions, Eur. Phys. J. C39 155 (2005) [hep-ph/0411040].

[4] A. Gehrmann-De Ridder et al., Photon plus jet-cross sections in deep inelastic e p collisions at order $O\left(\alpha^{2} \alpha_{S}\right)$, Nucl. Phys. B578 326 (2000) [hep-ph/0003082].

[5] M. Fontannaz et al., Isolated prompt photon photoproduction at NLO, Eur. Phys. J. C21 303 (2001) [hep-ph/0105121].

[6] A. V. Lipatov and N. P. Zotov, Prompt photon photoproduction at HERA in the $k(T)$-factorization approach, Phys. Rev. D72 054002 (2005) [hep-ph/0506044].
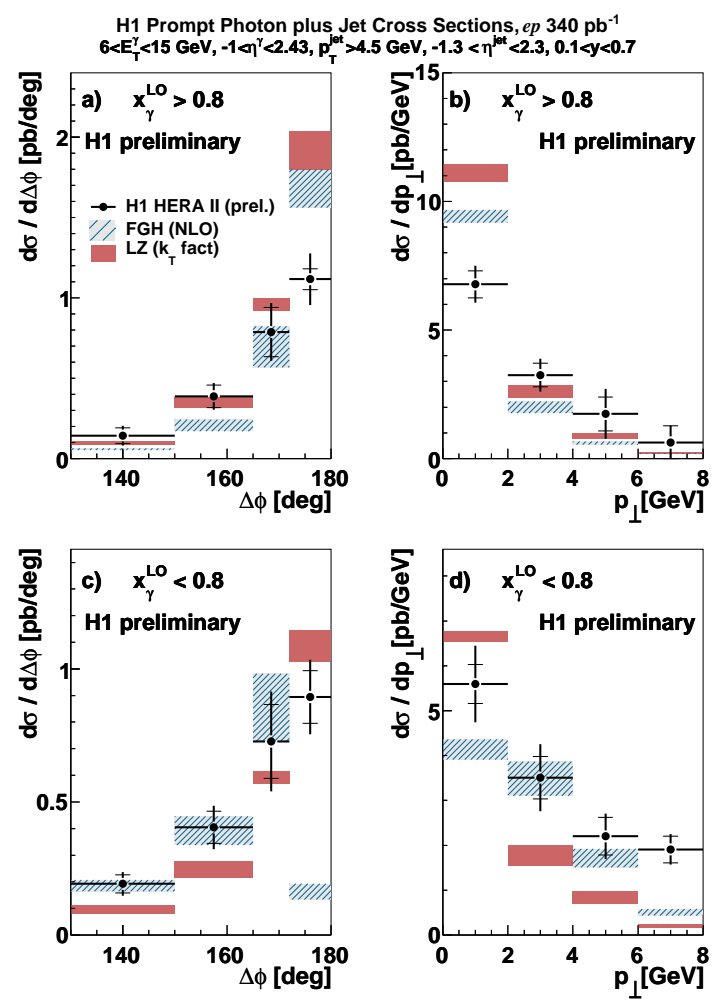

Figure 4: Preliminary differential prompt photon + jet cross section in photoproduction compared to the calculation predictions (see text) as measured by the $\mathrm{H} 1$ collaboration. 